\title{
Implementation of collective Thomson scattering on the TEXTOR tokamak for energetic ion measurements
}

\author{
L. Porte ${ }^{a)}$ \\ MIT Plasma Science and Fusion Center, Cambridge, Massachusetts 02139 \\ H. Bindslev \\ FOM Institute voor Plasmaphysica, EURATOM-Association, The Netherlands \\ F. Hoekzema \\ Forschungszentrum Jülich GmbH, Institut für Plasmaphysik, Germany \\ J. Machuzak and P. Woskov \\ MIT Plasma Science and Fusion Center, Cambridge, Massachusetts 02139 \\ D. Van Eester \\ Laboratory for Plasma Physics, Ecole Royale Militaire, Belgium
}

(Presented on 21 June 2000)

\begin{abstract}
Knowledge of the energy spectrum of fast ions in magnetically confined fusion plasmas is important and fundamental for achieving fusion energy production. Collective Thomson scattering (CTS) of high-power electromagnetic radiation is one of the most promising diagnostic methods for measuring the energy spectrum of confined fast ions. A CTS diagnostic has been implemented on the TEXTOR tokamak for the study of fast ions. It uses a $400 \mathrm{~kW}, 110 \mathrm{GHz}$ gyrotron source as the probe and a high-resolution heterodyne radiometer as the receiver. Details of the system are given in this article. First results from the diagnostic show that scattered radiation has been detected.
\end{abstract}

(C) 2001 American Institute of Physics. [DOI: 10.1063/1.1322617]

\section{INTRODUCTION}

In magnetically confined fusion plasma, nuclear reactions, ion cyclotron resonance heating (ICRH), and neutralbeam injection (NBI) generate fast ions. These fast ions sustain the nuclear reaction but can destabilize the plasma. One must measure the confined-fast-ion energy distribution to understand the fast-ion/plasma interaction. Collective Thomson scattering (CTS) is the most promising diagnostic technique for this purpose and has been applied successfully. Bindslev et al., ${ }^{1}$ using a $140 \mathrm{GHz}$ gyrotron, measured MeV-range fastion distributions on JET, while Behn et al., ${ }^{2}$ on TCA, and Suvorov et al. ${ }^{3}$ on W7-AS used $\mathrm{D}_{2} \mathrm{O}$ lasers $(385 \mu \mathrm{m})$ to measure the ion temperature.

Bindslev et al. ${ }^{4}$ report that TEXTOR is an excellent vehicle for the development and use of CTS. In this article, a $110 \mathrm{GHz}$ gyrotron scattering experiment that has been implemented on TEXTOR is described.

\section{RADIATION SOURCE}

A $110 \mathrm{GHz}$ gyrotron provides $\approx 400 \mathrm{~kW}$ of rf power. For CTS, the radiation is polarized to be in the $O$ mode. The rf power is fully modulated with a $50 \%$ duty cycle and a $\approx 7$ ms pulse length.

The gyrotron's output optics produce a beam that has $\approx 3 \mathrm{~cm}$ diam in the plasma center. The plasma-facing mirror can be rotated in the toroidal and poloidal planes, indepen-

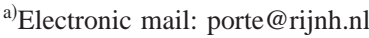

dently, and this permits scans in both position of the measurement volume and in the direction of the resolved velocity component.

Dobbe et al. ${ }^{5}$ will describe the gyotron in detail in a future publication.

\section{RECEIVER TRANSMISSION LINE AND ANTENNA}

To ensure sufficient spatial resolution, the overlap volume of the receive and probe beams must be kept sufficiently small. We require $\approx 10 \mathrm{~cm}$ radial resolution. Given the $3 \mathrm{~cm}$ diam of the probe beam, the receive beam is constrained to have an $H$-plane radius of $\approx 3 \mathrm{~cm}$ and an $E$-plane radius of $\approx 6 \mathrm{~cm}$ at a distance of $800 \mathrm{~mm}$ from the mirror.

An existing parabolic mirror of $50 \mathrm{~mm}$ focal length and $100 \mathrm{~mm}$ diam is used as the primary reflector in a center-fed reflector antenna. A simple rectangular feed horn has been designed, using the techniques of quasioptics, to produce the asymmetric beam described. The horn has internal dimensions $3.49 \mathrm{~mm}$ ( $H$ plane) by $3.37 \mathrm{~mm}$ ( $E$ plane) and is 7.55 $\mathrm{mm}$ long. Ideally, the horn would have been longer $(>12$ $\mathrm{mm})$ but space was very limited. The fundamental waveguide, at the horn's neck, is WR-8. The horn aperture is located on the mirror axis at a distance of $51.5 \mathrm{~mm}$. Details of the design are discussed by Porte et al. ${ }^{6}$ Figure 1 shows the measured variation of beam size as a function of distance from the mirror center.

The parabolic mirror can be rotated in the poloidal and toroidal directions on a shot-to-shot basis and its position has been calibrated. The WR- 8 guide at the neck of the feed horn selects $O$-mode polarization. The entire length of the 


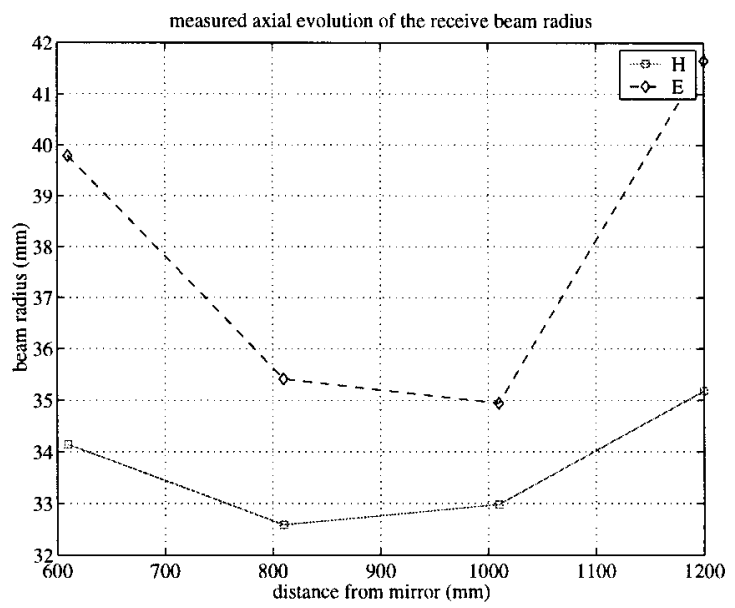

FIG. 1. Measured axial evolution of the beam size generated by the reflector antenna designed for the TEXTOR CTS diagnostic.

transmission line is $\approx 2 \mathrm{~m}$. An oversized waveguide is used for most of the run to reduce Ohmic losses. Immediately after the fundamental waveguide, at the neck of the feed horn, a waveguide transition leads to the WR-15 guide. This $\approx 1200$-mm-long, straight waveguide section carries the radiation, with little loss, to the receiver where another transition leads back to the fundamental guide. The vacuum seal is located in the WR-15 guide very close to the antenna mounting flange and is made of $100 \mu \mathrm{m}$ Kaptan film mounted between rubber $\mathrm{O}$ rings in an adapted UG-385/U waveguide flange. The antenna and transmission line, up to the receiver, has $\approx 10 \mathrm{~dB}$ attenuation.

\section{RECEIVER}

The receiver is a high-resolution heterodyne radiometer that has several features added to its front end as protection against stray gyrotron radiation. The receiver is housed in a copper box that provides physical protection and electromagnetic shielding. The layout of the receiver in its copper box is shown in Fig. 2. The receiver is made up of several components and these are described below.

Two rf notch filters, each with $\approx 60 \mathrm{~dB}$ attenuation, reject any stray gyrotron light. They have a $3 \mathrm{~dB}$ bandwidth of $\approx 400 \mathrm{MHz}$ and a notch width $\approx 150 \mathrm{MHz}$. Their passband is $10 \mathrm{GHz}$ on either side of $109.947 \mathrm{GHz}$. The notch filters

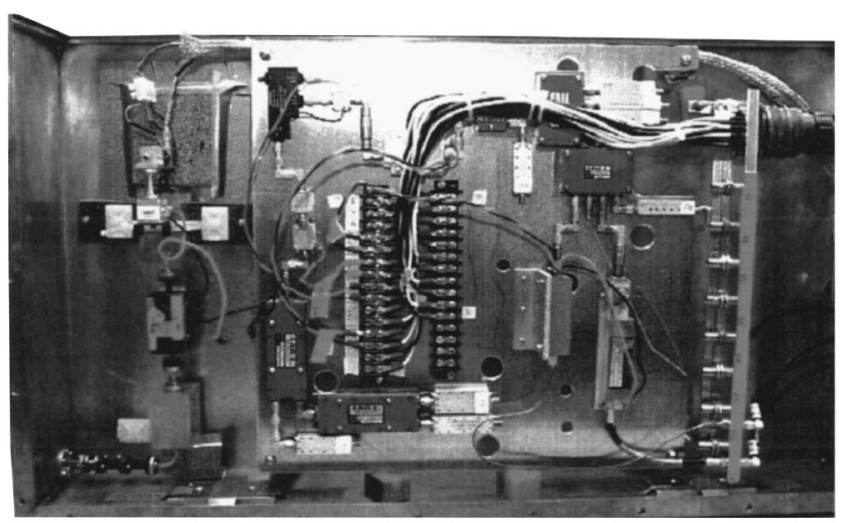

FIG. 2. Physical layout of the heterodyne receiver in its copper box.

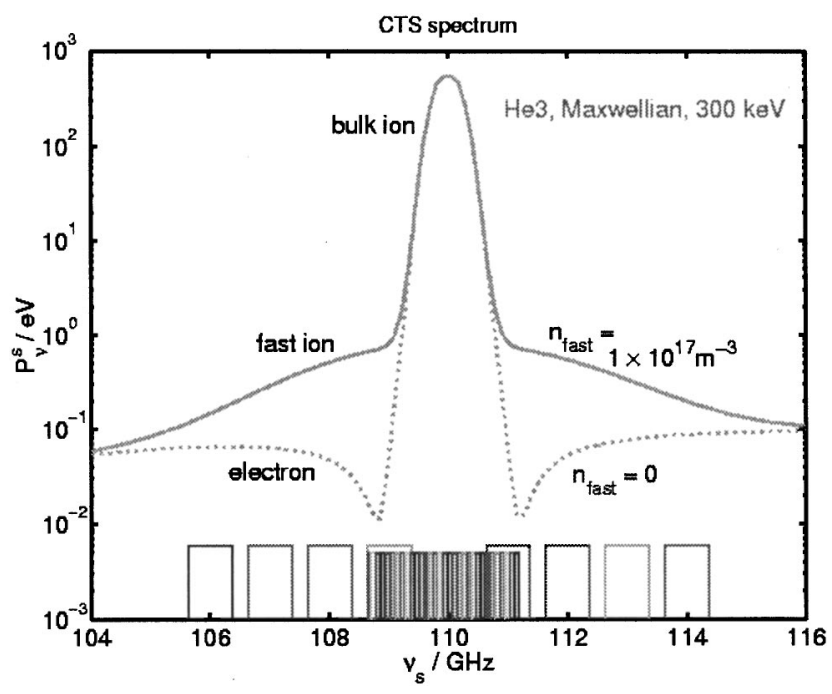

FIG. 3. CTS spectrum calculated assuming typical TEXTOR fast-ion parameters and scattering geometry. The vertical bars at the bottom of the graph show the location of the receiver's channels in the spectrum.

protect the mixer and IF amplifiers against $\approx 10 \mathrm{~mW}$ of stray gyrotron light and allow for $300 \mathrm{MHz}$ drift in the gyrotron frequency during each pulse. The rf notch filters are separated by a level set attenuator that provides padding and prevents a standing-wave effect between the two notch filters. An isolator would have been a better option for the prevention of standing wave, but this was impossible due to the receiver's proximity to the tokamak.

Immediately after the notch filter is a rf PIN switch that is used to reject radiation from spurious modes that occur as the gyrotron switches on and off. The PIN switch affords $\approx 20 \mathrm{~dB}$ rejection.

Following the rf PIN switch is the low-noise mixer and local oscillator. The local oscillator is a free-running Gunn diode oscillating at $100.5 \mathrm{GHz}$.

Immediately after the mixer is an IF triplexer that separates the lower sideband from the upper sideband and potential gyrotron radiation.

The separated IF signal is amplified by $\approx 30 \mathrm{~dB}$ in power in amplifiers whose noise figure is $\approx 2.5 \mathrm{~dB}$. These, in combination with the low-noise mixer, give the heterodyne radiometer a very low-noise temperature $\approx 0.1 \mathrm{eV}$. The IF amplifier gains are chosen to ensure signal levels remain $\approx 10$ $\mathrm{dB}$ removed from the amplifiers' $1 \mathrm{~dB}$ compression point to avoid modulated gain compression synchronous with the CTS signal.

Eight $750 \mathrm{MHz}$ bandwidth IF filters detect the fast-ion feature while $3280 \mathrm{MHz}$ bandwidth filters detect the central ion feature and part of the fast-ion feature. Each filter feeds a detector diode and dc amplifier. The output of 20 of the video amplifiers is digitized and stored on a networked workstation. Figure 3 shows the radiometer's spectral coverage of a calculated TEXTOR fast-ion spectrum.

The in situ receiver noise temperature is measured to be $<50 \mathrm{eV}$.

\section{PRELIMINARY RESULTS}

A series of commissioning and test experiments has been performed. 


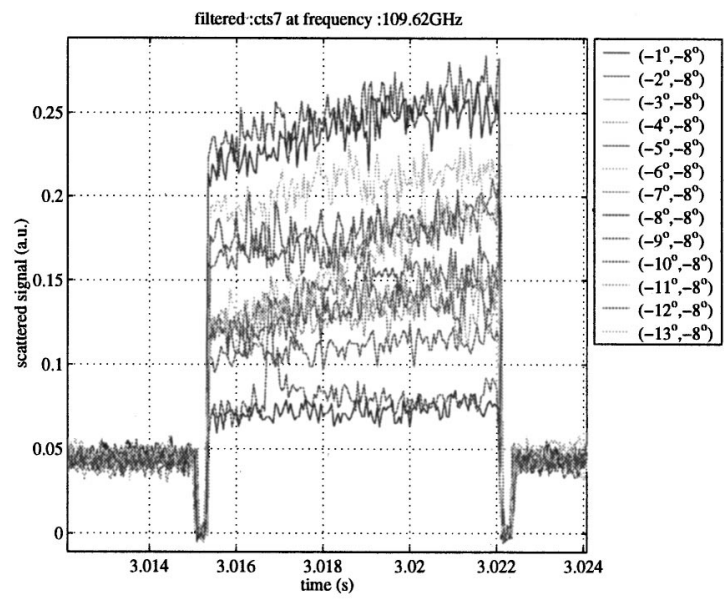

FIG. 4. Keeping the probe beam antenna in a fixed position, the receive antenna was rotated in a toroidal direction on a shot-to-shot basis. The level scattered signal was detected for each antenna orientation. The signal level reached a peak when the receive and probe antenna patterns overlapped. Here, the peak power is detected when the receive antenna is oriented at $-7^{\circ}$ and the probe antenna is at $-8^{\circ}$.

It is essential, to the performance of CTS, that the radiation source is spectrally pure. Considerable effort was put into ensuring that the gyrotron met this requirement and that its oscillation frequency was within the notch filters' rejection region.

Operation at a toroidal field of $2.6 \mathrm{~T}$ with NBI and ICRH heating has been established. It was decided to operate at 2.6 $\mathrm{T}$ to minimize the level of background millimeter-wave radiation by removing ECE resonances from the plasma. However, strong spurious signals, correlated with the gyrotron pulses with no receive/probe beam overlap were observed. Reducing the level of impurities in the plasma eliminated the spurious signals.

In an Ohmically heated plasma regime, with no spurious signals, an experiment was performed to detect scattered radiation. The probe antenna was kept in a fixed position while the receive antenna was translated in the toroidal direction. The level of signal, correlated with the gyrotron pulses, was measured. The level of correlated signals went through a maximum when the receive beam and probe beam were overlapping and diminished on either side. Figure 4 shows the scattered signal from one of the receiver's channels. The described trend is evident in the data. The peak scattered power was found when the receive antenna was oriented at a toroidal angle of $-7^{\circ}$. Figure 5 shows the variation of scattered signal, as a function of receive antenna orientation, for three different radiation frequencies. The angular width of the overlap region is consistent with the size of the overlap volume. A similar series of experiments has been performed in combined ICRH/NBI-heated plasmas and the results are very encouraging. When experiments are done with beam overlap, NBI-heated plasmas exhibit a broader spectrum with higher correlated signal than do Ohmically heated plasmas. These data are still being analyzed.

These observations are consistent with collective Thomson scattering.

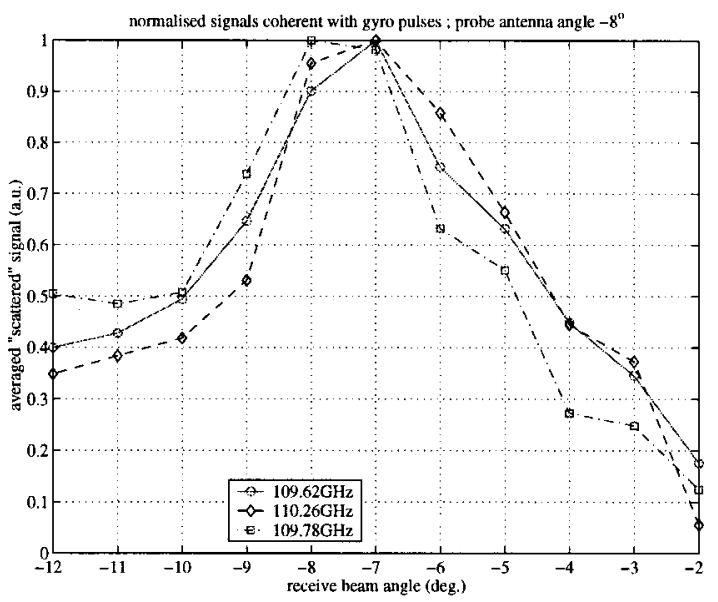

FIG. 5. With the probe radiation antenna in a fixed position the receive antenna was steered, from shot to shot, in a toroidal direction. The scattered signal reached a maximum at a position where the receive and probe beams were expected to overlap. The width of the overlap region is consistent with the size of the probe and receive antenna patterns. This experiment was done in Ohmic plasmas.

\section{CONCLUSIONS}

A CTS diagnostic, using a $110 \mathrm{GHz}, 400 \mathrm{~kW}$ gyrotron using $O$-mode polarization and a high spectral resolution heterodyne radiometer has been constructed and commissioned on TEXTOR tokamak. The gyrotron has been optimized for CTS and operates reliably and well. A receive antenna with an asymmetric antenna pattern has been designed and built and gives a radial spatial resolution of $\approx 10 \mathrm{~cm}$.

All aspects of the heterodyne receiver have been commissioned and are operational. The receiver has an estimated, in situ noise temperature $<50 \mathrm{eV}$.

A regime of operation has been established that will permit CTS experiments to be performed and initial plasma results show that a scattered signal has been detected. Physics experiments will commence toward the end of June 2000.

\section{ACKNOWLEDGMENTS}

The FOM Institute, EURATOM-Association; Forschungszentrum Jülich $\mathrm{GmbH}$; and the Ecole Royale Militaire are partners in the Trilateral EUREGIO cluster. This work was supported by the U.S. Dept. of Energy under Grant No. DE-FG02-94ER54325.

${ }^{1}$ H. Bindslev, J. A. Hoekzema, J. Egedal, J. A. Fessey, T. P. Hughes, and J. S. Machuzak, Phys. Rev. Lett. 83, 3206 (1999).

${ }^{2}$ R. Behn, D. Dicken, J. Hackman, S. A. Salito, M. R. Siegrist, P. A. Krug, I. Kjelberg, B. Duval, B. Joyce, and A. Pochelon, Phys. Rev. Lett. 62, 2833 (1989).

${ }^{3}$ E. V. Suvorov et al., Plasma Phys. Controlled Fusion 37, 1207 (1995).

${ }^{4}$ H. Bindslev, L. Porte, J. A. Hoekzema, J. S. Machuzak, P. Woskov, and D. Van Eester, Proceedings of the 26th Conference on Controlled Fusion and Plasma Physics, 14-16 June, Maastricht, The Netherlands (unpublished).

${ }^{5}$ N. Dobbe, M. Besterbreurtje, J. A. Hoekzema, O. G. Kruit, and R. Prins, (unpublished).

${ }^{6}$ L. Porte, H. Bindslev, J. S. Machuzak, and P. Woskov (unpublished). 